\title{
Editorial
}

\section{Advanced PHWR Safety Technology: PHWR Challenging Issues for Safe Operation and Long-Term Sustainability}

\author{
Jin Ho Song, ${ }^{1}$ Wei Shen, ${ }^{2}$ Malcolm Griffiths, ${ }^{3}$ Bo Wook Rhee, ${ }^{1}$ \\ YongMann Song, ${ }^{1}$ and Masanori Naitoh ${ }^{4}$ \\ ${ }^{1}$ Korea Atomic Energy Research Institute (KAERI), Daejeon 305-353, Republic of Korea \\ ${ }^{2}$ Canadian Nuclear Safety Commission (CNSC), Ottawa, ON, Canada K1P $5 S 9$ \\ ${ }^{3}$ Canadian Nuclear Laboratories (CNL), Chalk River, ON, Canada K0J $1 J 0$ \\ ${ }^{4}$ The Institute of Applied Energy (IAE), Tokyo 105-0003, Japan \\ Correspondence should be addressed to Jin Ho Song; dosa@kaeri.re.kr
}

Received 10 March 2015; Accepted 10 March 2015

Copyright (c) 2016 Jin Ho Song et al. This is an open access article distributed under the Creative Commons Attribution License, which permits unrestricted use, distribution, and reproduction in any medium, provided the original work is properly cited.

Pressurized heavy water reactor (PHWR) has been operated worldwide as one of the most efficient nuclear reactors owing to its superior neutron efficiency and flexibility to consider broad range of alternative fuel cycles. With the efforts for the life extension of the old reactors as well as the post-Fukushima safety enhancement, development of advanced PHWR safety technologies draws attention across the world. This issue on advanced PHWR safety technologies compiles 12 papers dealing with subjects such as moderator experiment and severe accident for PHWR, aging and plant life management, continued operation for PHWR, reactor physics and fuel performance technology for PHWR, and current PHWR safety R\&D status in Canada.

For moderator experiment, the research on the CANDU6 moderator circulation and related experiments including the scaling study, flow and temperature measurement techniques, and a CFD analysis are discussed.

For severe accident, the potential hazard of MCCI (molten corium-concrete interaction) with the uncertainties owing to the possibility of a calandria vault floor meltthrough is discussed. Also, based on the Fukushima experience, new safeguards have been proposed for PHWRs. The Fukushima accident is reviewed in terms of protection, electric power, and cooling. Recommendations include increasing the seismic rating of certain systems in addition to constructing external barriers for floods. The bunker concept enhances safety through electrical redundancy and diversity. The addition of an elevated external coolant tank increases the cooling duration time.

In the area of plant aging, mathematical fundamentals to deal with the pressure tube creep problem are formulated.

For reactor physics, as an alternative way to generate the two-group constants necessary for the CANDU-PHWR core analysis, utilizing B1-theory-augmented Monte-Carlo fewgroup-constant-generation method is proposed and successfully applied to CANDU-6 core analysis.

For fuel performance technology, CHF enhancement of advanced 37-element fuel bundles is discussed, in which two approaches to enlarge the flow areas of the peripheral subchannels of a center element are considered to investigate the impact of the flow area changes of the center subchannels on the CHF enhancement.

For the PHWR safety R\&D status, an overview of the CANDU safety R\&D activities at CANDU Owners Group (COG), Atomic Energy of Canada Limited (AECL), Candu Energy, Canadian Nuclear Safety Commission (CNSC), and Canadian Universities including SCWR activities, R\&D for aging reactors, and regulators oversight strategy against aging are discussed.

By compiling these papers, we hope to have a precious chance to share the results of R\&D efforts for the development of advanced PHWR technologies as well as to enrich our 
readers and researchers with respect to these emerging CANDU safety issues in the aftermath of the accidents at the Fukushima Daiichi nuclear power plant.

Jin Ho Song Wei Shen Malcolm Griffiths Bo Wook Rhee YongMann Song Masanori Naitoh 

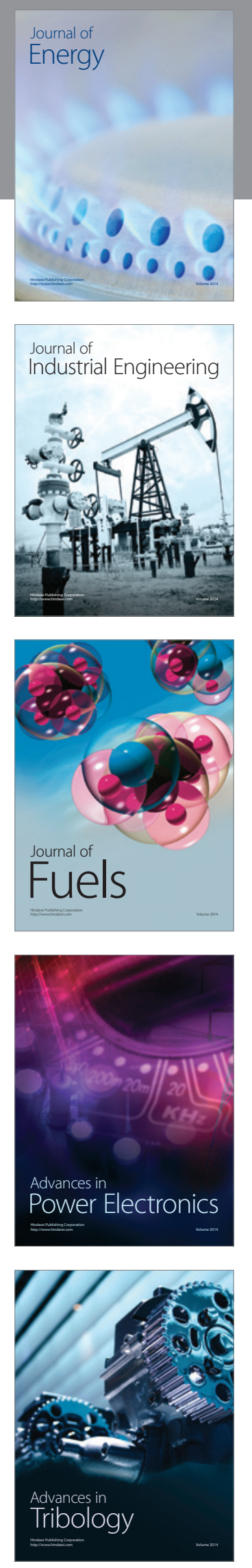


The Scientific World Journal


Submit your manuscripts at

http://www.hindawi.com
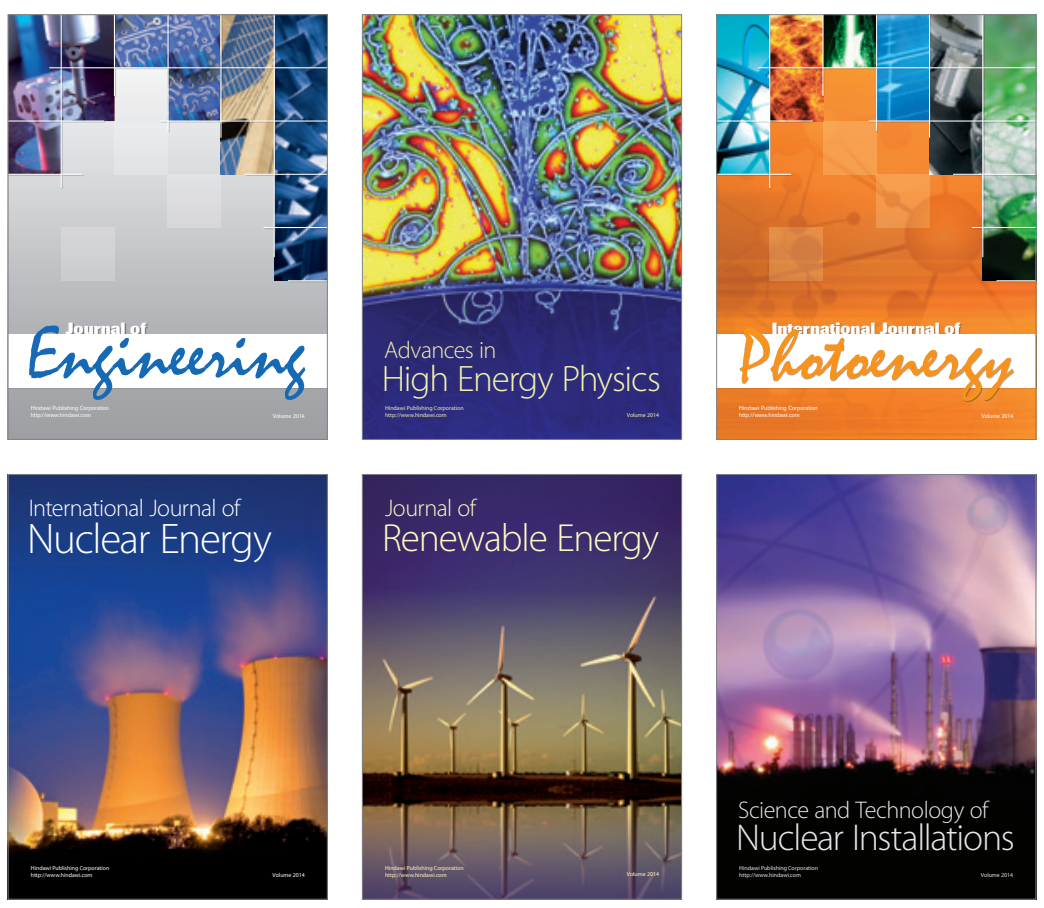
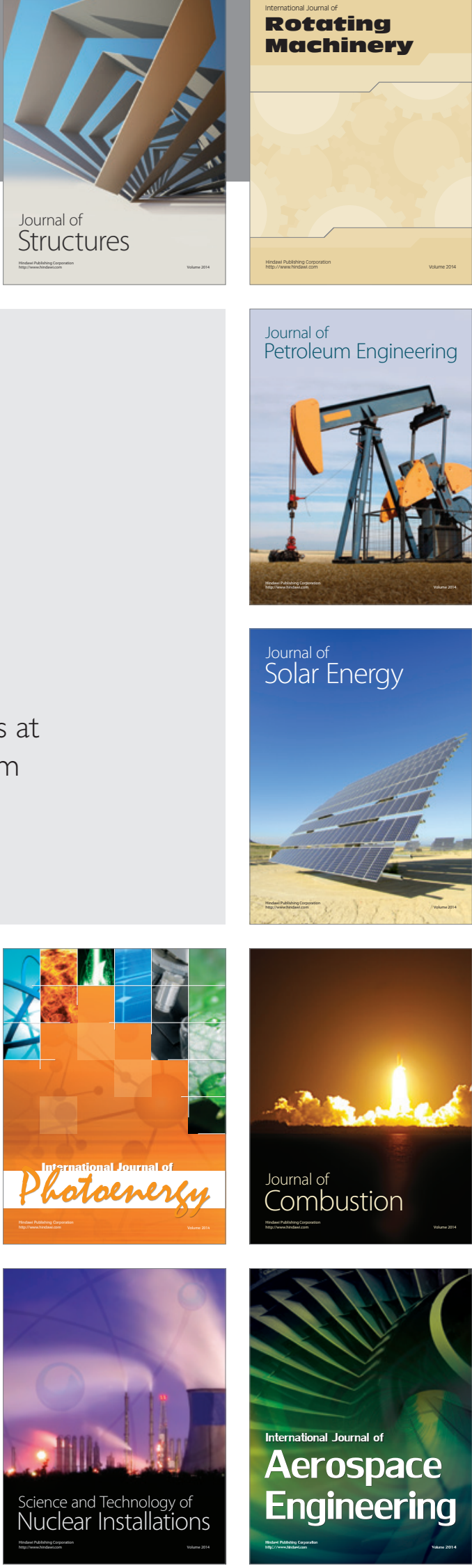